\title{
Soil Water Resource Use Limit by Plants
}

\author{
zhongsheng guo ${ }^{1}$ \\ ${ }^{1}$ State key laboratory of soil erosion and dry land farming on Loess Plateau, Institute of \\ Soil and Water Conservation, Northwestern A \& F University, Chinese Academy of Science
}

June 30, 2020

\begin{abstract}
Non-native vegetation with high canopy closure can alter the equilibrium relationship between soil water and plant growth into a non-equilibrium relationship leading to soil degradation, vegetation decline and crop failure in the most part of waterlimited regions. There is a need for the application of a novel theory in the assessment and the adjustment of non-equilibrium relationships to facilitate the sustainable management of non-native vegetation in the course of vegetation restoration in such regions. Therefore, a novel theory, Soil Water Resources Use Limit by Plant (SWRULP) has been developed. SWRULP refers to the amount of water stored in the maximum infiltration depth (MID) at which the soil moisture content in each layer is equivalent to the wilting coefficient. The wilting coefficient is expressed by the wilting coefficient of indicator plants in a plant community. SWRULP is an index for assessing plant overuse of soil water resources and the estimation of the starting time to regulate the relationship between soil water and plant growth in addition to the selection of the most suitable tree or plant species for vegetation restoration activities. To better understand SWRULP, in the present study, the SWRULP was assessed in a Caragana shrubland and an alfalfa grassland in semiarid loess hilly region. The results showed that the wilting coefficient varied with soil depth, and the SWRULP changed based on site conditions and vegetation type. When the soil water resources were equivalent to the SWRULP, soil water influenced plant growth considerably and caused the discoloration of sclerophyllous plants, abscission, cessation of growth, and soft leaf plant wilt. Under such conditions, the relationship between soil water and plant growth should be regulated based on soil water carrying capacity for vegetation to achieve sustainable use of soil water resource and sustainable management of forest and grassland and utilization, in addition to sustainable development.
\end{abstract}

\section{Hosted file}

SWRULP (2020) (Fig+Table). docx available at https://authorea.com/users/338284/articles/464329soil-water-resource-use-limit-by-plants 